\title{
AN EXACT SOLUTION FOR FLOW WITH CONDENSATION*
}

\author{
By MOSTAFA A. ABDELKADER (25 Sh. Champollion, Alexandria, Egypt)
}

1. Introduction. A nonlinear problem of flow of the vapor of a substance downward toward a cooling porous plate, which causes condensation, was considered by Yih [1]. The governing equations were reduced to the dimensionless forms (Yih's Eqs. (13) and (15)):

$$
\begin{gathered}
\theta+\frac{\gamma}{2}(\gamma-1) M^{2} V^{2}=-\theta^{\prime}-(\gamma-1) V, \\
\theta=\gamma M^{2}(V-1)\left(V+\sigma V^{\prime}\right)+V,
\end{gathered}
$$

where $\theta$ and $V$ denote variables related to the normalized temperature and the normalized velocity respectively, $\gamma$ is the ratio of the specific heats, $\sigma$ is a kind of Prandtl number, and $M$ is the Mach number far upstream. The primes denote differentiation with respect to $\eta$, which is proportional to distance from the vapor-liquid interface.

Assuming $M$ to be less than unity, Yih gave an approximate solution by expanding $\theta$ and $V$ in power series of $M^{2}$, and obtained explicit expressions for the first two terms of the expansions. No estimates for the errors of the approximate expressions were given.

Our purpose in this note is to present an exact solution in the $(\theta, V)$ phase plane when the parameters $\sigma$ and $M$ are certain functions of $\gamma$. This solution may thus be compared with Yih's approximate solution in order to estimate its accuracy for different values of $\gamma$ (and hence of $\sigma$ and $M$ ).

2. An exact solution. The phase-plane nonlinear differential equation, obtained from Eqs. (1) and (2), is

$$
\left.\left.\left\{\gamma M^{2} V(V-1)+V-\theta\right\} \frac{d \theta}{d V}=\sigma \gamma M^{2}(V-1)\right\}(\gamma-1) V+\frac{\gamma}{2}(\gamma-1) M^{2} V^{2}+\theta\right\} .
$$

We shall give the exact general solution of Eq. (3) for the case that $\sigma$ and $M$ are the following functions of $\gamma$ :

$$
\sigma=(3-\gamma) / 2 \gamma, \quad M^{2}=2 /(\gamma+3) .
$$

We introduce a new variable $y$ given by

$$
y=\theta+\left(\gamma M^{2}-1\right) V-\gamma M^{2} V^{2}
$$

which, using Eqs. (4), may be written

$$
y=\theta+\frac{\gamma-3}{\gamma+3} V-\frac{2 \gamma}{\gamma+3} V^{2}
$$

\footnotetext{
* Received June 25, 1980; revised version received August 7, 1980.
} 
From Eqs. (3), (4) and (5) we obtain

$$
y \frac{d y}{d V}+3 \frac{(1+\gamma)}{3+\gamma} y V=\frac{\gamma(3-\gamma)(1+\gamma)}{(3+\gamma)^{2}}\left(V-V^{3}\right) .
$$

Making in Eq. (6) the substitutions

$$
y=d x / d u, \quad x=(1+\gamma) /(2(3+\gamma)) V^{2},
$$

where $u$ is an auxiliary independent variable, we get the inhomogeneous linear differential equation

$$
\frac{d^{2} x}{d u^{2}}+3 \frac{d x}{d u}+2 \frac{\gamma(3-\gamma)}{1+\gamma} x=\frac{\gamma(3-\gamma)}{3+\gamma} .
$$

The general solution of Eq. (7) is well known and leads in a straightforward manner to that of Eq. (3) via $y$ and $x$. After some lengthy manipulations we find that the family of trajectories of Eq. (3), when $\sigma$ and $M$ satisfy Eqs. (4), is given by the one-parameter algebraic function:

$$
\left\{3(1-n) \xi-2 \gamma(3-\gamma)\left(V^{2}-1\right)\right\}^{1+n}=C\left\{3(1+n) \xi-2 \gamma(3-\gamma)\left(V^{2}-1\right)\right\}^{1-n},
$$

where

$$
\begin{aligned}
\xi & =2 \gamma V^{2}+(3-\gamma) V-(3+\gamma) \theta, \\
3 n & =\left|\frac{8 \gamma^{2}-15 \gamma+9}{\gamma+1}\right|^{1 / 2},
\end{aligned}
$$

and $C$ is a constant of integration.

From the definitions of $\theta$ and $V$ given by Eqs. (12) of [1], these are required to vanish far upstream, so that the single trajectory of interest in the problem is the one passing through the origin, for which $C$ is given by

$$
C=\{2 \gamma(3-\gamma)\}^{2 n} \text {. }
$$

\section{REFERENCE}

[1] C.-S. Yih, Flows with condensation, Quart. Appl. Math. 37, 401-409 (1980) 